\title{
Adenovirus-mediated expression of melanoma antigen gp75 as immunotherapy for metastatic melanoma
}

\author{
EA Hirschowitz ${ }^{1}$, S Leonard ${ }^{1}$, W Song ${ }^{1}$, B Ferris ${ }^{1}$, PL Leopold ${ }^{1}$, JJ Lewis ${ }^{2}$, WB Bowne ${ }^{2}$, S Wang ${ }^{2}$, \\ AN Houghton ${ }^{2}$ and RG Crystal ${ }^{1}$ \\ ${ }^{1}$ Division of Pulmonary and Critical Care Medicine, The New York Hospital-Cornell Medical Center, New York, NY; and \\ ${ }^{2}$ Department of Immunology, Memorial Sloan-Kettering Cancer Center, New York, NY, USA
}

\begin{abstract}
Melanocyte differentiation antigens, such as the brown locus protein gp75, are potential biological targets for immunotherapy. We investigated whether expression of the murine gp75 cDNA mediated by an adenovirus (Ad) vector could induce melanoma rejection using this model self antigen that usually induces tolerance, and whether Ad vector-directed production of interleukin-2 (IL2) might augment this response. To evaluate this approach, Ad vectors were constructed containing the murine gp75 cDNA (Ad.gp75) and the human IL2 cDNA (Ad.IL2). Efficacy was evaluated in C57BI/6 mice challenged i.v. with $10^{5} \mathrm{~B} 16$ cells, using the number of lung metastases as the efficacy parameter. Naive control mice developed $175 \pm 12$ metastases by day 14. Controls receiving intranasal Ad.IL2 1 day after B16 cell injection, intraperitoneal (i.p.) mitomycin-Ctreated B16 cells \pm i.p. Ad.IL2 before B16 cell challenge and Ad.ßgal-treated mice had similar numbers of meta-
\end{abstract}

stases as controls $(P>0.1)$. In marked contrast, preimmunization with intradermal Ad.gp75 provided dramatic reduction in the number of lung metastases (52 $\pm 7,29 \%$ of control). Addition of regional (intranasal delivery to the lung) Ad.IL2 to intradermal Ad.gp75 preimmunization 1 day following tumor challenge provided further protection (18 $\pm 6,10 \%$ of control). Depletion of $C D 4^{+}$and $C D 8^{+} T$ cell subsets effectively blocked the protective effect seen following immunization. Adoptive transfer of macrophagedepleted splenocytes from Ad.gp75-immunized mice similarly afforded significant protection against B16 tumor cell challenge. Further, serum obtained 21 days following Ad.gp75 immunization showed no detectable anti-gp75 antibody by immunoprecipitation. These results suggest that immunization with Ad.gp75 induces cellular immune responses that are capable of rejecting B16 melanoma in a host that is usually tolerant to gp75 antigen.

Keywords: adenovirus; vaccine; melanoma; gp75; IL2; antigen

\section{Introduction}

The immune response to human melanoma has been characterized in the past several years. ${ }^{1,2}$ Specific antigens identified in murine and human melanomas include MAGE-1 and MAGE-3, MART 1/melan-A, tyrosinase, gp100, mutated CDK4, a $97 \mathrm{kDa}$ cell surface glycoprotein melanotransferrin, a $66 \mathrm{kDa}$ protein and a $75 \mathrm{kDa}$ tyrosinase-related protein-1 (gp75). ${ }^{3-14}$ Based on extensive data in murine models and in human cancers showing that the immune system can recognize antigens expressed by melanoma, therapeutic strategies have been designed to augment immune recognition of melanoma antigens utilizing attenuated tumor cells or cell lysates, either alone or mixed with immune adjuvants, tumor cells genetically modified to express cytokines, specific antigenic peptides, or gene transfer vaccination approaches using cDNAs coding for specific antigens. ${ }^{15-47}$

Gp75 is one of the melanoma antigens that has been evaluated in protein-based vaccine strategies. ${ }^{41,48-51}$ The gp75 protein is an abundantly produced melanosome glycoprotein synthesized by pigmented melanocytes and

Correspondence: RG Crystal, Division of Pulmonary and Critical Care Medicine, The New York Hospital-Cornell Medical Center, 520 East 70th Street, ST505, New York, New York 10021, USA

Received 16 October 1997; accepted 4 February 1998 melanomas but not in other cells. ${ }^{48,52}$ The protein is an integral part of the melanosome membrane and has been identified as inducing antigen-specific $\mathrm{B}$ cell and $\mathrm{T}$ cell responses in individuals with melanoma. ${ }^{51-53}$ Human gp75 has been purified and identified as the homologue of the mouse $b$ (brown) locus gene product. ${ }^{49,50,54}$ Similar to human gp75, murine gp75 is overexpressed in some murine melanomas. ${ }^{50}$ The present study is directed toward the hypothesis that adenovirus (Ad) vectormediated transfer of the murine gp75 cDNA can induce sufficient immunity in naive syngeneic $\mathrm{C} 57 \mathrm{Bl} / 6$ mice to suppress the growth of B16 melanoma cells in lung following intravenous challenge with the tumor. Based on the knowledge that Ad-mediated in vivo transfer of transgenes often evokes both a cellular and a humoral immune response against the transgene, ${ }^{55-60}$ we have constructed an Ad vector expressing the murine gp75 cDNA (Ad.gp75), and used this vector to immunize mice against syngeneic tumor expressing gp75. The data demonstrate this strategy is effective, appears to be dependent on cell-mediated immunity, and the antitumor immunity evoked by Ad.gp75 is further augmented by regional expression of interleukin-2 (IL2) delivered with an Ad vector. 

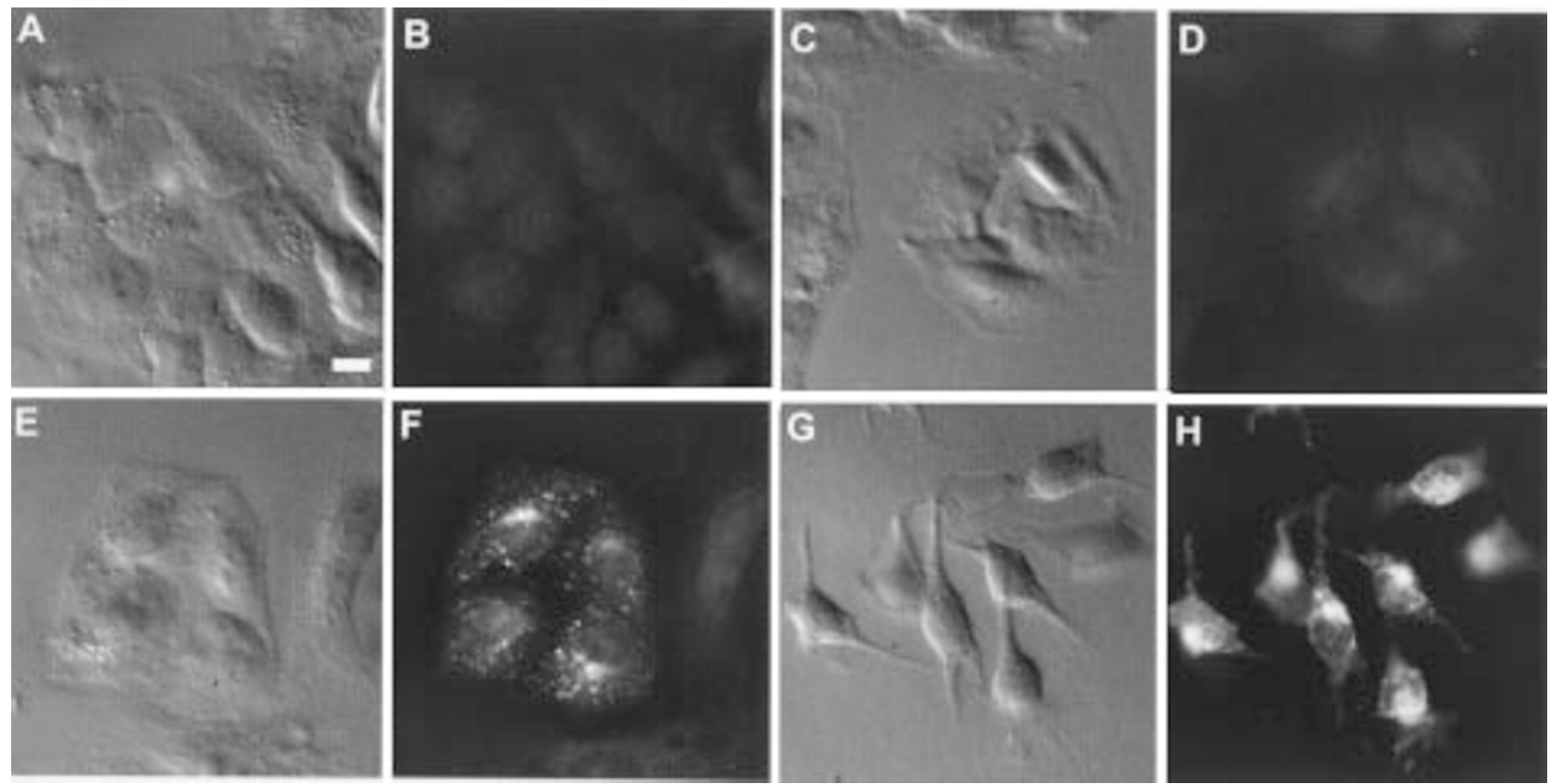

Figure 1 Expression of gp75 following in vitro infection with Ad.gp75. Human A549 lung adenocarcinoma cells were infected with Ad.gp75 (MOI 10); after $48 \mathrm{~h}$ the cells were evaluated for expression of gp75 using a gp75-specific monoclonal antibody and FITC-labeled second antibody. (A and B) Uninfected A549 cells. (C and D) A549 cells infected with control virus. (E and F) A549 cells infected with Ad.gp75. (G and H) B16 melanoma cells, which endogenously express the gp75 protein, as a positive control. ( $A, C, E$ and $G$ ) Differential interference contrast microscopy. (B, D, F and $H$ ) Immunofluorescence. Contrast enhancement to $(B, D$ and $F)$ are identical. The maximum fluorescence intensity in panel $H$ was 2.6 times greater than (E) in the unmodified images. Bar $=10 \mu \mathrm{m}$.

\section{Results}

In vitro expression of gp75 protein

To evaluate the ability of Ad.gp75 to produce a gp75 protein in target cells, human A549 lung adenocarcinoma cells were infected with Ad.gp75 multiplicity of infection (MOI) 10 or control virus (MOI 10), with uninfected A549 cells serving as a negative control, and B16 murine melanoma cells known to express gp75 endogenously as a positive control. Immunostaining with an anti-gp75 antibody revealed a high level of Ad.gp75 protein in gp75infected A549 cells, the expected expression of gp75 in the B16 cells, and no expression in cells infected with a control virus or uninfected cells (Figure 1). Fields from treated and control groups were imaged using both differential interference contrast microscopy and epifluorescence microscopy.

\section{In vivo regional Ad-directed IL2 production}

To evaluate the level of IL2 production in the lung following Ad.IL2 regional delivery, $\mathrm{C} 57 \mathrm{Bl} / 6$ mice received a single transnasal dose of Ad.IL2 $\left(10^{9}\right.$ p.f.u.; $\left.n=5\right)$ or control virus $\left(10^{9}\right.$ p.f.u.; $\left.n=3\right)$ transnasally and IL $2 / \mathrm{mg}$ lung protein was evaluated at $48 \mathrm{~h}$, with naive mice serving as negative control. The Ad.IL2 vector yielded $3.8 \pm 0.8 \mu \mathrm{g}$ IL2/mg lung protein, whereas delivery of control virus yielded undetectable levels of IL2, similar to naive mice. To control for the theoretical variable of neutralizing immunity generated by the host in the setting of prior Ad.gp75 immunization, Ad.gp75 (109 p.f.u.) was delivered (by the intranasal route as in subsequent experiments) 7 days before Ad.IL2 administration. After $48 \mathrm{~h}$, the IL2 levels in the lung were $4.3 \pm 0.5 \mu \mathrm{g}$ IL2/mg protein, similar to Ad.IL2 alone $(P>0.1)$ (Figure 2). There was insignificant systemic leak of IL2 in Ad.IL2treated compared with naive mice measured by IL2 ELISA in the serum $(0.2 \pm 0.1 \mu \mathrm{g}$ IL $2 / \mathrm{ml}$ serum compared with control $0.1 \pm 0.08 \mu \mathrm{g} \mathrm{IL} 2 / \mathrm{ml} ; P>0.1)$. There was no

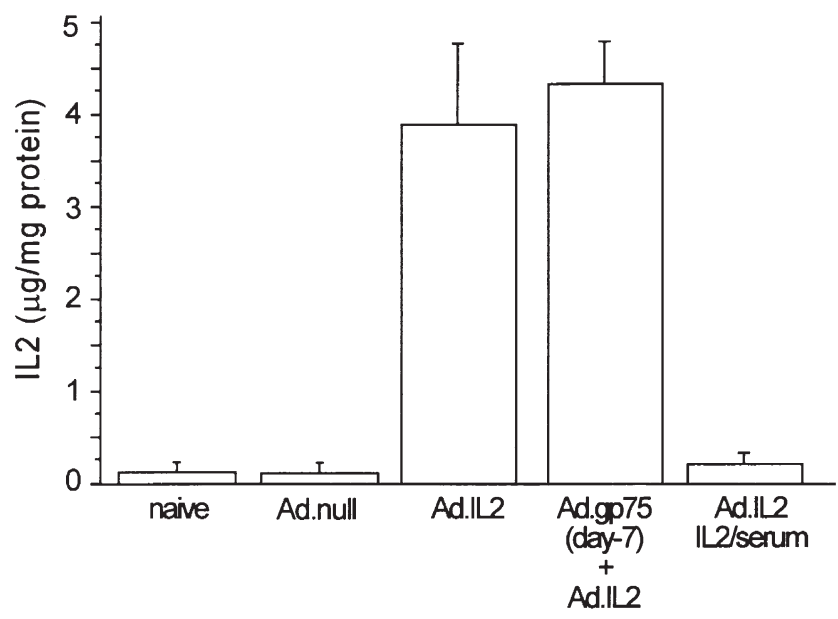

Figure 2 Ad-directed IL2 expression in vivo. Ad.IL2 or control virus AdCMV. Bgal (both $10^{9}$ p.f.u.) were delivered transnasally to the respiratory epithelium of C57Bl/6 mice; after $48 \mathrm{~h}$, IL2 levels were quantified by ELISA in the lungs of mice. To control for the theoretical variable of reduced gene transfer secondary to neutralizing immunity following Ad.gp75 immunization, Ad.gp75 (10 p.f.u.) was administered intradermally 7 days before administration of Ad.IL2 (day -7); after 48 h, IL2 levels were quantified and compared with naive controls. The data are expressed as $\mu \mathrm{g} I L 2 / m g$ protein in the various groups. The data represent mean \pm standard error of the mean of three animals per group in two independent experiments. 
toxicity associated with IL2 expression in the lungs determined by no increase in mortality over 2 weeks after vector administration.

\section{Effect of Ad-mediated regional delivery of IL2 against pulmonary metastases}

Mice receiving i.v. administration of B16 cells were treated 1 day and 5 days after injection with Ad.IL2 (10 ${ }^{9}$ p.f.u.) transnasally, and the tumor load was evaluated at 14 days as the number of pulmonary metastases on the surface of the lung. Quantification showed regional delivery and expression of IL2 provided no therapeutic advantage when compared with control ( $P>0.1$; Figure 3$)$.

\section{Protective effect of Ad.gp75 immunization \pm Ad.IL2}

The ability of the Ad.gp75 vector to induce immunity directed against B16 cells was evaluated in a similar fashion as for Ad.IL2. Naive control mice developed $175 \pm 12$ metastases by day 14 . In mice previously receiving a live tumor vaccine with B16 murine melanoma cells treated with mitomycin $C$, there was no significant protection against subsequent i.v. tumor challenge (reflected as the number of pulmonary metastases; $P>0.1$ compared with naive animals) (Figure 4). B16 cells treated with mitomycin $\mathrm{C}$ delivered intraperitoneally (i.p.) in concert with i.p. Ad.IL2 (109 p.f.u.) offered no significant protection $(P>0.1)$. Similarly, Ad. $3 g a l$ had no protective effect $(P>0.1)$. In contrast, a single intradermal immunization with Ad.gp75 7 days before tumor cell challenge provided marked protection against tumor cell challenge, reflected as a decrease in the number of metastases to $29 \pm 4 \%$ of the number seen in naive controls $(P<0.01)$. The addition of regional Ad-directed IL2 expression within the lung provided additional significant protec-

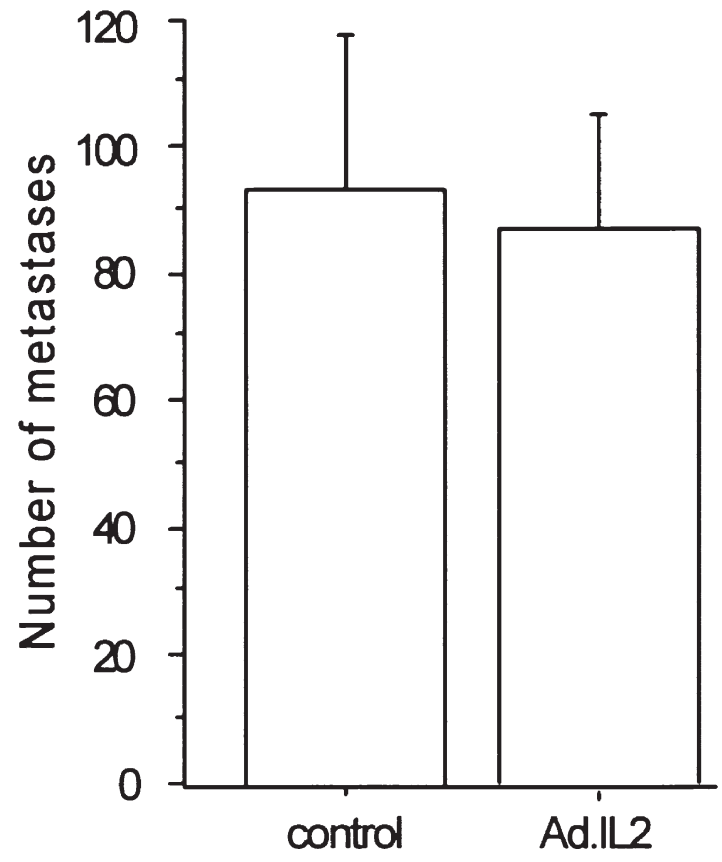

Figure 3 Effect of regional delivery of Ad.IL2 on B16 tumor growth into the lungs. B16 tumor cells $\left(10^{5}\right)$ were administered i.v. to C57Bl/6 mice and $24 \mathrm{~h}$ later, Ad.IL2 ( $10^{9}$ p.f.u.) was administered to the respiratory epithelium. The data are expressed as mean \pm standard error of the mean of the number of pulmonary metastases in treatment and control mice with five mice in each group.

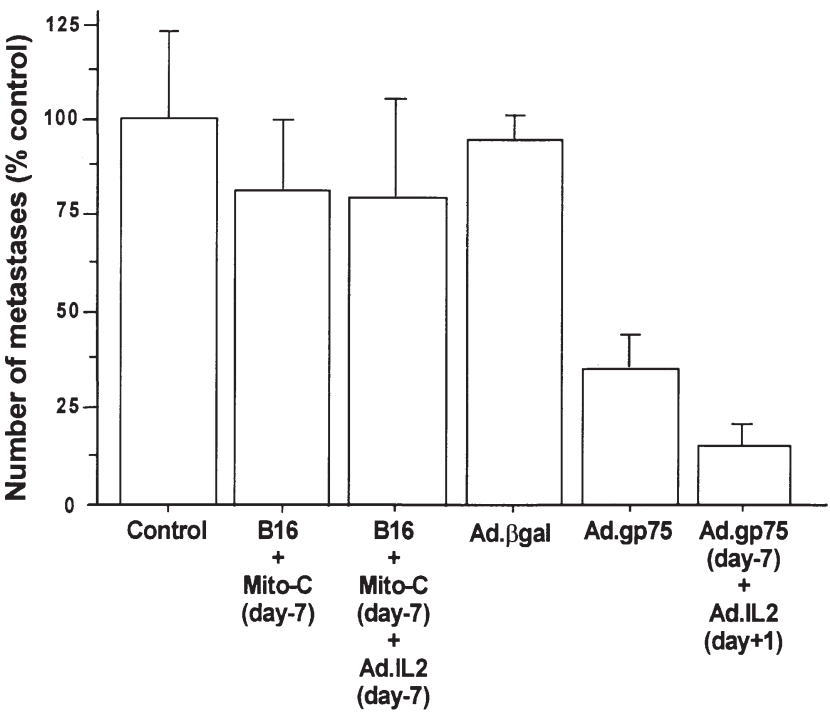

Figure 4 Protective immunity induced by Ad.gp75 alone and in combination with regional delivery of Ad.IL2. C57Bl/6 mice were immunized with Ad.gp75 (109 p.f.u., ID) 7 days before $($ day -7$)$ i.v. challenge with syngeneic B16 murine melanoma cells (105 i.v.). In a subgroup of animals, Ad.IL2 (109 p.f.u.) was administered to the respiratory epithelium 1 day following tumor challenge $($ day +1$)$. Experimental groups include naive control; i.p. immunization with mitomycin C-treated B16 cells 7 days before tumor challenge; i.p. immunization with mitomycin C-treated B16 cells + i.p. Ad.IL2 (109 p.f.u.) 7 days before tumor challenge; ID administration of Ad. $\beta g a l$ (109 p.f.u.); ID immunization with Ad.gp75 (10 p.f.u.) 7 days before tumor challenge; and mice immunized ID with Ad.gp75 $\left(10^{9}\right.$ p.f.u.) 7 days before tumor challenge and treated with intranasal

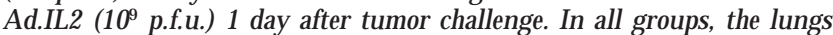
were evaluated for the number of tumor nodules 14 days after tumor challenge. The data represent four separate experiments, each compared with its own naive control (minimum $n=8$ animals per group), and are expressed as percentage of control.

tion to Ad immunization alone $(10 \pm 4 \%$ of control; $P<0.01$ compared with naive; $P<0.01$ compared with Ad.gp75 alone). As a further measure of tumor burden in Ad.gp75-immunized mice, comparisons were made with the number of metastases seen following dose escalating B16 tumor cell challenge. These mice showed a roughly linear increase in the number of metastases following B16 cell challenge between $10^{4}$ and $10^{5}$ cells, thus as expected the tumor burden in mice following Ad.gp75 immunization was significantly less than $5 \times 10^{5}$ cells $(P<0.05)$ and similar to a tumor cell challenge of $2.5 \times 10^{4}$ B16 cells $(P>0.1)$, and in mice treated with a combination of Ad.gp75 and Ad.IL2 was similar to $10^{4}$ to $2.5 \times 10^{4}$ B16 cells $(P>0.1$ both comparisons; not shown).

\section{Mechanisms of protective immunity}

To explore the dependence of the tumor immunization on $\mathrm{T}$ cells, Ad.gp75-immunized mice were selectively depleted of either $\mathrm{CD}^{+}$or $\mathrm{CD}^{+} \mathrm{T}$ cells and assessed for their ability to reject B16 tumor cell challenge. Selective depletion was confirmed by cytofluorometric analysis on pooled residual splenocytes. The protective Ad.gp75 immunization was abolished, with a similar number of metastases developing in mice receiving anti-CD4 or antiCD8 antibodies compared with control $(P>0.1$; Figure 5). This is surprising, since only two-thirds of cells were shown to be depleted at the time of death. An isotype control had no significant effect on the protection 


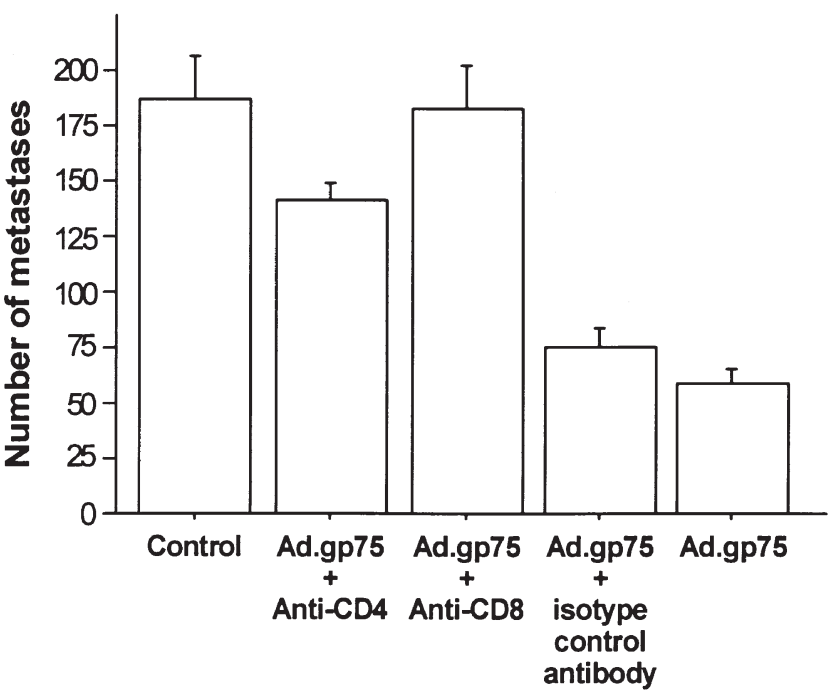

Figure 5 Selective depletion of $\mathrm{CD}^{+}$or $\mathrm{CD} 8^{+} \mathrm{T}$ cell subsets following immunization with Ad.gp75. Mice were immunized ID as described for Figure 4 and subgrouped to receive either anti-CD4, anti-CD8 monoclonal antibodies, isotype control monoclonal antibody, or no additional treatment. Mice were challenged with $10^{5}$ B16 cells i.v. as above and the effect of $T$ cell depletion was compared with naive control and Ad.gp75-immunized animals with intact cellular immunity.

afforded by Ad.gp75 immunization $(P>0.1$ compared with Ad.gp75 immunization). To confirm the importance of cellular immunity in the suppression of B16 pulmonary metastases, splenocytes were harvested from mice immunized with Ad.gp75 7 days before or naive mice, depleted of macrophages, and $10^{7}$ cells from either naive or immunized mice were adoptively transferred to naive mice which were then challenged with B16 tumor cells at $24 \mathrm{~h}$. Consistent with cell-mediated mechanisms, mice receiving splenocytes from Ad.gp75-immunized mice developed only $43 \pm 14 \%$ of metastases seen in naive controls $(57 \%$ reduction; $P<0.01)$. Mice receiving control splenocytes showed a similar number of metastases to naive control $(P>0.1$; Figure 6). Importantly, no evidence of humoral immunity against gp75 was detected in serum taken from mice 21 days after a single administration of Ad.gp75 using immunoprecipitation, determined by specific binding to $S^{35}$-methionine-labeled B16 lysates, whereas positive control TA99 antibody yielded the predicted $75 \mathrm{kDa}$ band. This method has been shown sensitive enough to detect the presence of anti-gp75 autoantibodies which correlates with antitumor effects seen in mice immunized with human gp75 protein or murine gp75 protein expressed in insect cells. ${ }^{41}$ Consistent with these findings, immunization with syngeneic gp75 protein did not induce auto-antibodies to gp $75 .{ }^{41}$

\section{Discussion}

The current study evaluates the potential of immunotherapy directed toward metastatic melanoma using adenovirus-mediated in vivo delivery of the cDNA encoding the wild-type murine gp75 melanoma-associated antigen to syngeneic mice. Using the number of pulmonary metastases as a measure of tumor burden, the data show a single intradermal injection of the Ad vector Ad.gp75 effectively protects mice against intravenous

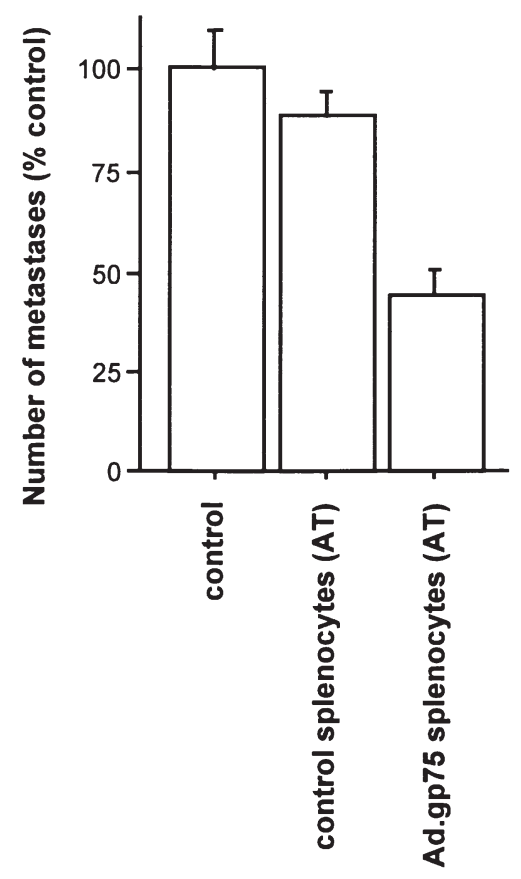

Figure 6 Adoptive transfer of splenocytes from Adgp75-immunized mice to naive mice before B16 tumor cell challenge. Mice were immunized ID as above and splenocytes harvested at day 7. Purified splenocytes were plated in vitro for $2 \mathrm{~h}$ to remove adherent macrophages and adoptively transferred by tail vein to naive mice at $10^{7}$ cells per mouse. Twenty-four hours following mononuclear cell adoptive transfer, mice were challenged with $10^{5}$ B16 cells i.v. The protection afforded by the transfer was measured as reduction in pulmonary metastasis at 14 days after tumor cell challenge and is expressed as percentage of control. AT, adoptive transfer.

challenge of murine B16 melanoma cells which express gp75. Depletion of $\mathrm{CD}^{+}$and $\mathrm{CD}^{+}$lymphocytes abbrogated this antitumor effect suggesting an integral role for cell-mediated immunity. The suggested mechanism of cell-mediated immunity was further supported by the finding that adoptively transferred splenocytes from Ad.gp75-immunized mice to naive mice also afforded a high level of protection against subsequent tumor cell challenge. Consistent with these observations the antitumor effect was augmented regionally by the addition of Ad-mediated delivery of the human IL2 cDNA to the lungs of immunized mice. In contrast, Ad.IL2 offered no therapeutic advantage without prior Ad.gp75 immunization.

\section{gp75 Antigen in immunotherapy}

The human and murine gp75 antigen (also referred to as the tyrosinase-related protein-1; TRP-1) are related 75 $\mathrm{kDa}$ glycoproteins found in mice on the murine brown locus. ${ }^{41,48-51,54}$ Both human and murine gp75 are abundant in pigmented melanocytes and nevi, primary melanoma, and metastatic melanoma (melanosomal origin), and are not expressed in other human tissues. Gp75 was originally identified in association with high affinity IgG auto-antibodies in a patient with melanoma, 53,61 and has subsequently been shown to induce a specific $\mathrm{T}$ cell response which is HLA-A31 restricted. ${ }^{51}$ The potential of 
the gp75 antigen for immunotherapy is suggested by the finding that a modified form of the murine gp75 protein, as well as the human gp75 protein, possessing $80.2 \%$ homology to the murine gp75, is effective at inducing protective immunity against B16-derived pulmonary metastases. ${ }^{41}$ Interestingly, the unmodified wild-type murine gp75 protein has not been shown to induce protective immunity. ${ }^{41}$ Other than gp75, a variety of melanoma antigens have been identified including MAGE-1 and MAGE-3, MART 1/Melan-A, tyrosinase, gp100 and mutated CDK4, and are currently being evaluated in peptide-based, or vector-delivered gene-based vaccination trials. ${ }^{39,47}$

\section{Ad-mediated transfer of the gp75 cDNA}

Adenovirus-mediated gene-based vaccines take advantage of the high efficiency of gene transfer in vivo of this vector and capitalize on the host cellular immune responses known to be directed against Ad vector transgenes. ${ }^{55-60,62,63}$ This paradigm has been evaluated using vectors carrying genes for a variety of foreign proteins; in these studies in vivo delivery of recombinant Ad vectors generates immunity against xenogenized tumor cells expressing the foreign antigen leading to rejection of the modified tumor cells. ${ }^{62}$ Ad-mediated delivery of the human melanoma antigen gp100 cDNA can effectively immunize mice against murine tumors expressing murine gp100, which has a high degree of homology with the human gp100. ${ }^{46}$ The current study extends this paradigm by showing Ad-mediated in vivo delivery of an unmodified autologous cDNA is capable of inducing protective immunity breaking tolerance against a tumor expressing the autologous antigen. The finding that Ad.gp75, carrying the unmodified wild-type murine gp75 cDNA, is capable of effectively vaccinating syngeneic mice against challenge of B16 melanoma expressing the autologous gp75 is in contrast to the finding that wild-type gp75 peptide vaccination does not appear to be effective. ${ }^{41}$ The reasons the Ad vector gene-based gp75 vaccine is capable of inducing protective immunity to an autologous antigen remain to be evaluated, but differences from peptide-based vaccines may result from the protracted expression of antigen following gene delivery in the setting of an inflammatory response known to result from Ad vector infection. ${ }^{57,64}$ The kinetics of gene expression, optimal dosing and route of administration of the vector, the potential for repeated administration, as well as comparative information on gene delivery of various isoforms of the gp75 antigen (modified murine versus human gp75) remain to be evaluated.

\section{Augmentation of Ad.gp75 immunization with regional delivery of Ad.IL2}

Although single administration of Ad.gp75 was capable of protecting against a subsequent tumor burden by $68 \%$ compared with control, some tumor burden remains in these animals. In the context that cellular mechanisms appear to be invoked (as demonstrated by the response to depletion of $\mathrm{CD}^{+}$or $\mathrm{CD} 8^{+}$lymphocytes), the addition of IL2 is intended to supplement the stimulatory signals to effector cells, thus leading to further activation and clonal expansion of antitumor cellular immune processes. ${ }^{65,66}$ To approach this concept, we attempted to avoid the toxicity associated with systemically administered IL2, ${ }^{67,68}$ by delivering IL2 directly to the target organ, thus limiting systemic side-effects. The vector Ad.IL2 produced high levels of IL2 in the area to which the vector was directed, but showed no significant systemic leak of IL2 and animals all appeared healthy throughout the 2 week treatment period. Although some studies have shown ex vivo modification of tumor cells with the IL2 CDNA $^{26,69,70}$ or that in vivo viral vectormediated production of IL2 by established tumor can inhibit tumor growth, ${ }^{71-77}$ regional delivery of Ad.IL2 to the respiratory epithelium alone offered no significant therapeutic benefit against pulmonary metastasis to mice receiving an intravenous tumor burden. This therapy was, however, able to complement Ad-mediated gp75 gene expression, providing further significant protection against tumor challenge. In this context, Ad-mediated gene-based gp75 vaccines together with regional Ad vector-delivered IL2 may be a useful adjuvant to current therapy for limited disease for prevention of pulmonary metastasis.

\section{Materials and methods}

\section{Adenovirus vectors}

The E1a-, partial E1b ${ }^{-}$, partial E3- Ad vectors Ad.gp75 and Ad.IL2 were constructed using the Ad5 pJM17 backbone recombination plasmid pNY.102 (for Ad.gp75), and the recombination plasmid pCMV.S2+ for Ad.IL2. ${ }^{78}$ The Ad.gp75 vector expression cassette (right to left orientation) contains the cytomegalovirus immediateearly (CMV) promoter, followed by an artificial splice sequence, the murine gp75 cDNA, and the SV40 polyadenylation sequence. The Ad.IL2 vector is identical except for the human interleukin 2 (IL2) cDNA (gift of Bernard Gansbacher, Memorial Sloan-Kettering Cancer Center, New York, NY, USA) in the expression cassette in a left to right orientation in place of the gp75 cDNA. Both vectors were plaque purified, amplified, purified and titrated as previously described..$^{79,80}$ The control vectors Ad.null, similar to therapeutic vectors but carrying no transgene, and Ad.ßgal expressing the E. coli $\beta$-galactosidase ( $\beta$ gal) gene, were amplified as previously described..$^{78}$

\section{Ad.IL2 production of human IL2 in vivo}

To test Ad.IL2 directed expression of human IL2, Ad.IL2 $\left(10^{9}\right.$ p.f.u.; $\left.n=5\right)$ or control virus Ad $\beta$ gal $\left(10^{9}\right.$ p.f.u.; $\left.n=3\right)$ were delivered transnasally to the respiratory epithelium of C57BL/6 mice. To control for the theoretical variable of reduced gene transfer secondary to neutralizing immunity following Ad.gp75 immunization that could potentially limit expression of Ad.IL2, ${ }^{59}$ Ad.gp75 (10 ${ }^{9}$ p.f.u.) was delivered intradermally 7 days before IL2 delivery $(n=3)$. At $48 \mathrm{~h}$, the lungs were removed, homogenized in protein lysis buffer $(10 \mathrm{~mm}$ Tris- $\mathrm{HCl}, 2 \%$ Triton X-100, $0.025 \%$ sodium azide, $0.14 \mathrm{~m} \mathrm{NaCl}, 1 \mathrm{~mm}$ phenylmethanesulfonyl fluoride, $30 \mu \mathrm{m}$ leupeptin, $30 \mu \mathrm{m}$ apoprotinin, $30 \mu \mathrm{m}$ peptistatin) (Sigma, St Louis, MO, USA) using a Polytron homogenizer. Total protein was quantified using a BioRad protein assay (BioRad, Hercules, CA, USA), and IL2 tissue levels were quantified per mg protein using an enzyme-linked immuno assay (ELISA; R\&D, Minneapolis, MN, USA) and compared with naive control. To assess whether there was significant systemic leak of IL2 following regional delivery of Ad.IL2 to the lungs, blood was taken from the above ani- 
mals at the time of death by right heart puncture and the amount of IL2 per ml serum was assessed by ELISA as described above.

\section{Immunostaining for Ad-mediated gp75 transfer in vitro}

To evaluate the expression of gp75 antigen in transduced target cells, A549 human lung adenocarcinoma cells (ATCC, Rockville, MD, USA) were infected with Ad.gp75 (MOI 10). Negative controls include uninfected cells and A549 cells infected with Ad.ßgal (MOI 10). B16 murine melanoma cells endogenously expressing gp75 served as a positive control. The cells $\left(10^{5}\right)$ were infected in suspension and plated (on a chamber slide) for $48 \mathrm{~h}$ and fixed with $4 \%$ paraformaldehyde. The paraformaldehyde was inactivated with $5 \mathrm{~mm} \mathrm{NH} \mathrm{mH}_{4} \mathrm{CL}$, the cells permeabilized with $0.01 \%$ saponin with $1 \%$ bovine serum albumin in

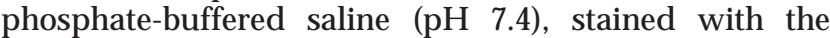
TA99 anti-gp75 monoclonal antibody (1:200 dilution), and counterstained with a fluorescein isothiocyanate (FITC)-conjugated goat anti-mouse secondary antibody. ${ }^{81}$ Representative fields were imaged with a Nikon Microphot SA, equipped with a $\times 40,0.7$ NA objective lens, Nikon FITC epifluorescence filter cube, and differential interference contrast optics (Nikon, Melville, NY, USA). Images were collected with a cooled chargecoupled device camera (Princeton Instruments, Trenton, NJ, USA).

\section{Cell line and animal model}

The B16 murine melanoma cell line is a weakly immunogenic tumor which expresses the gp75 protein in melanosomes within the cells and on the cell surface. ${ }^{61}$ The cell line is syngeneic to the $\mathrm{C} 57 \mathrm{Bl} / 6$ mouse and reliably produces pulmonary metastases following i.v. injection. ${ }^{82}$ To establish metastases, $10^{5} \mathrm{~B} 16$ cells were injected by the lateral tail vein to C57Bl/ 6 mice (Charles River, Wilmington, MA, USA). The numbers of metastases were quantified by counting the black tumor nodules on the surface of the lung. ${ }^{82}$

\section{In vivo delivery of Ad.IL2 to the lungs of tumor bearing mice}

Mice were challenged with B16 tumor $\left(10^{5}\right.$ i.v.) followed 1 day later by Ad.IL2 to the respiratory epithelium $\left(10^{9}\right.$ p.f.u. via nasal instillation; $n=4)$, or no therapy $(n=5)$, and repeated following a 5 day interval. Mice were killed 14 days following tumor cell delivery and the numbers of pulmonary metastases were counted under a dissecting microscope in both treatment and control (naive) groups.

\section{Suppression of pulmonary metastasis using adenovirus-} mediated immune modulation

Treatment groups include mice immunized intradermally (ID) 7 days before tumor challenge ('day -7 ') with Ad.gp75 (109 p.f.u.) with no additional therapy $(n=9)$, or Ad.gp75 plus regional administration of Ad.IL2 to the respiratory epithelium 1 day following tumor challenge ( $10^{9}$ p.f.u. via nasal instillation; $\left.n=10\right)$. Control groups included naive $\mathrm{C} 57 \mathrm{Bl} / 6$ mice, mice immunized with B16 cells treated with mitomycin C (Sigma, St Louis, MO, USA; $5 \times 10^{5}$ cells i.p., day -7), mitomycin C-treated B16 cells followed by Ad.IL2 (10 p.f.u., i.p., day -7$)$ or mice immunized with Adßgal $\left(10^{9}\right.$ p.f.u., ID, day -7). Lungs were removed 14 days after tumor cell challenge and the number of tumor nodules were counted under a dissecting microscope. The data represent the combination of four independent experiments each with independent controls (minimum $n=8$ animals per experiment). To quantify further the protective effect of this therapy, a titration of B16 tumor cells was performed to compare the relative tumor burden seen in Ad.gp75 immunized mice with an equivalent tumor cell challenge. Control naive mice received an i.v. injection of $10^{5}, 5 \times 10^{4}, 2.5 \times 10^{4}$, and $10^{4}$ B16 cells as described and the numbers of tumor nodules were evaluated at day 14 .

\section{In vivo depletion of $T$ cell subsets}

Ad.gp75-immunized mice were selectively depleted of $\mathrm{CD}^{+}$or $\mathrm{CD}^{+}$lymphocytes using monoclonal antibodies ( $n=7$ each group) and compared with immunized mice receiving no antibody $(n=20)$ and naive control $(n=16) .{ }^{61}$ Mice received i.p. injections of $200 \mu \mathrm{g}$ anti-CD4 (GK1.5, TIB-207 hybridoma, LT3T4; ATCC) anti-CD8 (TIB-105 hybridoma, Lyt-2; ATCC) or isotype control antibody ( IgG $_{2 \mathrm{~A}}$ к; PharMingen, San Diego, CA, USA) 2 days before and 2, 7 and 12 days after tumor cell challenge. Mice were evaluated for selective depletion of targeted lymphocyte populations by cytofluorometric analysis (Coulter, Miami, FL, USA). Residual spleen cells pooled from three mice from each group were stained with FITC-conjugated anti-mouse CD3a antibody, PEconjugated anti-mouse CD8e or conjugated anti-mouse CD4 antibody, or a combination of anti-CD3/anti-CD4 or anti-CD3/anti-CD8 (Pharmingen). At the time of death there was a $65 \%$ reduction in the number of $\mathrm{CD}^{+} 1 \mathrm{ym}-$ phocytes following treatment with anti-CD4 antibody compared with mice immunized with Ad.gp75, and a $66 \%$ reduction in the number of $\mathrm{CD}^{+}$lymphocytes following treatment with anti-CD8 antibody compared with naive, and a 73 and $74 \%$ reduction compared with mice immunized with Ad.gp75, respectively.

\section{Adoptive transfer of mononuclear splenocytes}

Mice were immunized with Ad.gp75 as described above. At 7 days, mononuclear splenocytes from immunized and naive mice were isolated as previously described. ${ }^{60}$ Five spleens from each group were harvested fresh and placed in a tissue culture dish with complete Dulbecco's modified Eagle's medium (DMEM; Biofluids, Rockville, MD, USA). The spleens were minced and ground, sheared with a 19-gauge needle, passed through a $200 \mu \mathrm{m}$ mesh to remove fibrous tissue. Cells were pelleted then resuspended in DMEM plus 10\% fetal bovine serum (Gibco BRL, Grand Island, NY, USA), and 50 units $/ \mathrm{ml}$ penicillin $\mathrm{G}, 50 \mu \mathrm{g} / \mathrm{ml}$ streptomycin (Gibco BRL) ('complete media'; $5-8 \times 10^{7}$ cells per spleen). Live lymphocytes were separated from dead cells and RBCs using a Ficoll-Paque (Pharmacia, Piscataway, NJ, USA) density separation technique, washed, then resuspended in complete media. Pooled splenic mononuclear cells from each group were plated in tissue culture for $2 \mathrm{~h}$ to allow macrophages to adhere to the plate. Nonadherent cells from each group were removed and resuspended at $10^{7}$ cells / $200 \mu \mathrm{l}$ PBS, then adoptively transferred to naive mice via tail vein injection. At $24 \mathrm{~h}$ mice were challenged with $10^{5}$ B16 melanoma cells i.v. as previously described. Tumor burden was evaluated at 14 days by counting the number of pulmonary nodules as described above. 
Detection of serum anti-gp75 antibodies

Mice were immunized with $10^{9}$ p.f.u. Ad.gp75 ID and serum collected 21 days following immunization and tested by immunoprecipitation as previously described. ${ }^{83}$ B16 cells $\left(5 \times 10^{6}\right)$ were preincubated in methionine-free medium $\left(3 \mathrm{~h}, 37^{\circ} \mathrm{C}\right)$ then metabolically labeled with $300 \mu \mathrm{Ci} \quad{ }^{35} \mathrm{~S}$-methionine $/ 10^{7}$ cells (specific activity $1140 \mathrm{Ci} / \mathrm{mmol} ; 7.9 \mathrm{mCi} / \mathrm{ml}$; New England Nuclear, Boston, MA, USA; $18 \mathrm{~h}, 37^{\circ} \mathrm{C}$ ). Cells were washed and solubilized at $10^{7}$ cells $/ \mathrm{ml}$ in lysis buffer $(150 \mathrm{~mm} \mathrm{NaCl}, 10 \mathrm{~mm}$ Tris- $\mathrm{HCl}, \mathrm{pH} 7.6,1 \% \mathrm{NP}-40,2 \mathrm{~mm}$ ethylenediaminetetraacetate (EDTA); $\left.30 \mathrm{~min}, 4^{\circ} \mathrm{C}\right)$. Serum $(5 \mu \mathrm{l})$ or TA99 $(1 \mu \mathrm{l}) \mathrm{MAb}$ was incubated with $150 \mu \mathrm{l}$ lysate at $4^{\circ} \mathrm{C}$ for $30 \mathrm{~min}$. Immune complexes were recovered on $30 \mu \mathrm{l}$ protein A bound agarose beads (Pharmacia, Piscataway, NJ, USA) washed (15 mm NaCl, $10 \mathrm{~mm}$ Tris- $\mathrm{HCl}, \mathrm{pH}$ 7.6, 1\% NP-40, $5 \mathrm{~mm}$ EDTA) and eluted with Laemmli buffer (0.01 m Tris- $\mathrm{HCl} \mathrm{pH} 7.2,2 \%$ sodium dodecyl sulfate (SDS), $12.0 \mathrm{mg} / \mathrm{ml}$ dithiotreol, $15 \%$ (w/v) sucrose, $0.01 \%$ pyronin $\mathrm{Y} ; 5 \mathrm{~min}, 100^{\circ} \mathrm{C}$ ). Samples were analyzed on a $10 \%$ SDS-polyacrylamide gel. Radioactive bands were revealed by fluorography.

\section{Statistical analysis}

All data are presented as mean \pm standard error of the mean and all comparisons were made using the unpaired two-tailed Student's $t$ test.

\section{Acknowledgements}

We would like to thank Hassan Naama and Carlo Russo for their helpful discussions, and N Mohamed for help in preparation of the manuscript. These studies were supported, in part, by the National Cancer Institute 1R01CA75192-01; by the Will Rogers Memorial Fund, White Plains, NY; GenVec, Inc., Rockville, MD; and NIH grant CA56821, Swim Across America, The Louis and Anne Abrons Foundation, and the Milstein Family Fund by the National Cancer Institute 1R01CA75192-01.

\section{References}

1 Mukherji B, Chakraborty NG. Immunobiology and immunotherapy of melanoma. Curr Opin Oncol 1995; 7: 175-184.

2 Rankin EM. Scientific aspects of gene therapy in melanoma. Curr Opin Oncol 1995; 7: 192-196.

3 Real FX et al. Class 1 (unique) tumor antigens of human melanoma: identification of unique and common epitopes on a 90kDa glycoprotein. Proc Natl Acad Sci USA 1988; 85: 3965-3969.

4 van der Bruggen $\mathrm{P}$ et al. A gene encoding an antigen recognized by cytolytic T lymphocytes on a human melanoma. Science 1991; 254: 1643-1647.

5 Traversari $\mathrm{C}$ et al. A nonapeptide encoded by human gene MAGE-1 is recognized on HAL-A1 by cytolytic T lymphocytes directed against tumor antigen MZ2-E. J Exp Med 1992; 176: 1453-1457.

6 Brichard $\mathrm{V}$ et al. The tyrosinase gene codes for an antigen recognized by autologous cytolytic T lymphocytes on HLA-A2 melanomas. J Exp Med 1993; 178: 489-495.

7 Zakut $\mathrm{R}$ et al. Differential expression of mage- $1,-2$, and -3 messenger RNA in transformed and normal human cell lines. Cancer Res 1993; 53: 5-8.

8 Bakker $\mathrm{AB}$ et al. Melanocyte lineage-specific antigen gp100 is recognized by melanoma-derived tumor-infiltrating lymphocytes. J Exp Med 1994; 179: 1005-1009.

9 Gaugler B et al. Human gene mage-3 codes for an antigen recognized on a melanoma by autologous cytolytic $\mathrm{T}$ lymphocytes. J Exp Med 1994; 179: 921-930.
10 Kawakami $\mathrm{Y}$ et al. Cloning of the gene coding for a shared human melanoma antigen recognized by autologous $\mathrm{T}$ cells infiltrating into tumor. Proc Natl Acad Sci USA 1994; 91: 35153519.

11 Kawakami $\mathrm{Y}$ et al. Identification of a human melanoma antigen recognized by tumor-infiltrating lymphocytes associated with in vivo tumor rejection. Proc Natl Acad Sci USA 1994; 91: 6458-6462.

12 van der Bruggen $P$ et al. A peptide encoded by human gene mage- 3 and presented by HLA-A2 induces cytolytic T lymphocytes that recognize tumor cells expressing mage-3. Eur J Immunol 1994; 24: 3038-3043.

13 Wolfel T et al. A p16ink4a-insensitive CDK4 mutant targeted by cytolytic T lymphocytes in a human melanoma. Science 1995; 269: 1281-1284.

14 Fleischhauer $\mathrm{K}$ et al. Multiple HLA-A alleles can present an immunodominant peptide of the human melanoma antigen melan-a/mart-1 to a peptide-specific HLA-A*0201+ cytotoxic T cell line. I Immunol 1996; 157: 787-797.

15 Livingston PO et al. Vaccines containing purified GM2 ganglioside elicit GM2 antibodies in melanoma patients. Proc Natl Acad Sci USA 1987; 84: 2911-2915.

16 Estin CD et al. Recombinant vaccinia virus vaccine against the human melanoma antigen p97 for use in immunotherapy. Proc Natl Acad Sci USA 1988; 85: 1052-1056.

17 Morton DL et al. Active specific immunotherapy in malignant melanoma. Semin Surg Oncol 1989; 5: 420-425.

18 Bystryn JC. Tumor vaccines. Cancer Metastasis Rev 1990; 9: 81-91.

$19 \mathrm{Kahn} \mathrm{M}$ et al. $\mathrm{CD}^{+} \mathrm{T}$ cell clones specific for the human p97 melanoma-associated antigen can eradicate pulmonary metastases from a murine tumor expressing the p97 antigen. J Immunol 1991; 146: 3235-3241.

20 McGee JM. Immunotherapy for malignant melanoma: a review and update. Semin Surg Oncol 1991; 7: 217-220.

21 Morton DL et al. Prolongation of survival in metastatic melanoma after active specific immunotherapy with a new polyvalent melanoma vaccine. Ann Surg 1992; 216: 463-482.

22 Chapman PB, Houghton AN. Non-antibody immunotherapy of cancer. Curr Opin Immunol 1993; 5: 726-731.

23 Crowley NJ, Seigler HF. Possibilities of immunotherapy and gene therapy for malignant melanoma. Semin Surg Oncol 1993; 9: 273-278.

24 Pardoll DM. New strategies for enhancing the immunogenicity of tumors. Curr Opin Immunol 1993; 5: 719-725.

25 Helling $F$ et al. $G_{\mathrm{D} 3}$ vaccines for melanoma: superior immunogenicity of keyhole limpet hemocyanin conjugate vaccines. Cancer Res 1994; 54: 197-203.

26 Tepper RI, Mule JJ. Experimental and clinical studies of cytokine gene-modified tumor cells. Hum Gene Ther 1994; 5: 153-164.

27 Aichele $\mathrm{P}$ et al. $\mathrm{T}$ cell priming versus $\mathrm{T}$ cell tolerance induced by synthetic peptides. J Exp Med 1995; 182: 261-266.

28 Bakker $\mathrm{AB}$ et al. Generation of antimelanoma cytotoxic T lymphocytes from healthy donors after presentation of melanomaassociated antigen-derived epitopes by dendritic cells in vitro. Cancer Res 1995; 55: 5330-5334.

29 Barth A, Morton DL. The role of adjuvant therapy in melanoma management. Cancer 1995; 75: 726-734.

30 Hoon DS, Yuzuki D, Hayashida M, Morton DL. Melanoma patients immunized with melanoma cell vaccine induced antibody responses to recombinant mage-1 antigen. J Immunol 1995; 154: 730-737.

31 Kitamura $\mathrm{K}$ et al. Serological response patterns of melanoma patients immunized with a gm2 ganglioside conjugate vaccine. Proc Natl Acad Sci USA 1995; 92: 2805-2809.

32 Miller $\mathrm{K}$ et al. Improved survival of patients with melanoma with an antibody response to immunization to a polyvalent melanoma vaccine. Cancer 1995; 75: 495-502.

33 Morioka $\mathrm{N}$ et al. Cytotoxic $\mathrm{T}$ cell recognition of a human melanoma derived peptide with a carboxyl-terminal alanine-proline sequence. Mol Immunol 1995; 32: 573-581.

34 Mukherji B et al. Induction of antigen-specific cytolytic T cells in situ in human melanoma by immunization with synthetic peptide-pulsed autologous antigen presenting cells. Proc Natl Acad Sci USA 1995; 92: 8078-8082. 
35 Parmiani G, Colombo MP. Somatic gene therapy of human melanoma: preclinical studies and early clinical trials. Melanoma Res 1995; 5: 295-301.

36 Dalgleish A. The case for therapeutic vaccines. Melanoma Res 1996; 6: 5-10.

$37 \mathrm{Hu} \mathrm{X}$ et al. Enhancement of cytolytic T lymphocyte precursor frequency in melanoma patients following immunization with the MAGE-1 peptide loaded antigen presenting cell-based vaccine. Cancer Res 1996; 56: 2479-2483.

38 Jaeger $\mathrm{E}$ et al. Generation of cytotoxic $\mathrm{T}$ cell responses with synthetic melanoma-associated peptides in vivo: implications for tumor vaccines with melanoma-associated antigens. Int J Cancer 1996; 66: 162-169.

39 Maeurer MJ, Storkus WJ, Kirkwood JM, Lotze MT. New treatment options for patients with melanoma: review of melanomaderived $\mathrm{T}$ cell epitope-based peptide vaccines. Melanoma Res 1996; 6: 11-24.

40 Mastrangelo MJ et al. Active specific immunization in the treatment of patients with melanoma. Semin Oncol 1996; 23: 773-781.

41 Naftzger $C$ et al. Immune response to a differentiation antigen induced by altered antigen: a study of tumor rejection and autoimmunity. Proc Natl Acad Sci USA 1996; 93: 14809-14814.

42 Parkhurst MR et al. Improved induction of melanoma-reactive CTL with peptides from the melanoma antigen gp100 modified at HLA-A*0201-binding residues. I Immunol 1996; 157: 25392548.

43 Rosenberg SA. The immunotherapy of solid cancers based on cloning the genes encoding tumor-rejection antigens. Annu Rev Med 1996; 47: 481-491.

44 Salgaller ML, Marincola FM, Cormier JN, Rosenberg SA. Immunization against epitopes in the human melanoma antigen gp100 following patient immunization with synthetic peptides. Cancer Res 1996; 56: 4749-4757.

45 Yee $\mathrm{C}$ et al. Isolation of tyrosinase-specific $\mathrm{CD} 8^{+}$and $\mathrm{CD} 4^{+} \mathrm{T}$ cell clones from the peripheral blood of melanoma patients following in vitro stimulation with recombinant vaccinia virus. J Immunol 1996; 157: 4079-4086.

46 Zhai Y et al. Antigen-specific tumor vaccines. Development and characterization of recombinant adenoviruses encoding mart1 or gp100 for cancer therapy. I Immunol 1996; 156: 700-710.

47 Rosenberg SA. Phase I trial in patients with metastatic melanoma of immunization with a recombinant adenovirus encoding the MART-1 melanoma antigen. Office of Recombinant DNA Activities: National Institutes of Health, 1995, RAC No. 9512-140.

48 Vijayasaradhi S, Doskoch PM, Houghton AN. Biosynthesis and intracellular movement of the melanosomal membrane glycoprotein gp75, the human b (brown) locus product. Exp Cell Res 1991; 196: 233-240.

49 Vijayasaradhi S, Houghton AN. Purification of an autoantigenic 75-kDa human melanosomal glycoprotein. Int J Cancer 1991; 47: 298-303.

50 Vijayasaradhi S, Bouchard B, Houghton AN. The melanoma antigen gp75 is the human homologue of the mouse b (brown) locus gene product. J Exp Med 1990; 171: 1375-1380.

51 Wang RF et al. Identification of a gene encoding a melanoma tumor antigen recognized by HLA-A31-restricted tumor-infiltrating lymphocytes. J Exp Med 1995; 181: 799-804.

52 Tai T, Eisinger M, Ogata S, Lloyd KO. Glycoproteins as differentiation markers in human malignant melanoma and melanocytes. Cancer Res 1983; 43: 2773-2779.

53 Mattes MJ, Thomson TM, Old LJ, Lloyd KO. A pigmentationassociated, differentiation antigen of human melanoma defined by a precipitating antibody in human serum. Int J Cancer 1983; 32: 717-721.

54 Yokoyama K, Yasumoto K, Suzuki H, Shibahara S. Cloning of the human dopachrome tautomerase/tyrosinase-related protein 2 gene and identification of two regulatory regions required for its pigment cell-specific expression. J Biol Chem 1994; 269: 27080-27087.

55 Juillard $\mathrm{V}$ et al. Long-term humoral and cellular immunity induced by a single immunization with replication-defective adenovirus recombinant vector. Eur J Immunol 1995; 25: 34673473.

56 Van Ginkel FW et al. Intratracheal gene delivery with adenoviral vector induces elevated systemic IgG and mucosal IgA antibodies to adenovirus and beta-galactosidase. Hum Gene Ther 1995; 6: 895-903.

57 Tripathy SK, Black HB, Goldwasser E, Leiden JM. Immune responses to transgene-encoded proteins limit the stability of gene expression after injection of replication-defective adenovirus vectors. Nat Med 1996; 2: 545-550.

58 Yang $\mathrm{Y}$ et al. Immune responses to viral antigens versus transgene product in the elimination of recombinant adenovirusinfected hepatocytes in vivo. Gene Therapy 1996; 3: 137-144.

59 Mack CA et al. Circumvention of anti-adenovirus neutralizing immunity by administration of an adenoviral vector of an alternate serotype. Hum Gene Ther 1997; 8: 99-109.

60 Song W, Kong H-L, Traktman P, Crystal RG. Cytotoxic T lymphocyte responses to proteins encoded by heterologous transgenes transferred in vivo by adenovirus vectors. Hum Gene Ther 1997; 8: 1207-1217.

61 Hara I, Takechi Y, Houghton AN. Implicating a role for immune recognition of self in tumor rejection: passive immunization against the brown locus protein. J Exp Med 1995; 182: 1609-1614.

62 Chen PW et al. Therapeutic antitumor response after immunization with a recombinant adenovirus encoding a model tumorassociated antigen. I Immunol 1996; 156: 224-231.

63 Ragot $\mathrm{T}$ et al. Replication-defective recombinant adenovirus expressing the Epstein-Barr virus (EBV) envelope glycoprotein gp340/220 induces protective immunity against EBV-induced lymphomas in the cottontop tamarin. J Gen Virol 1993; 74: 501-507.

64 Yang Y, Wilson JM. Clearance of adenovirus-infected hepatocytes by MHC class I-restricted $\mathrm{CD} 4^{+} \mathrm{CTLs}$ in vivo. J Immunol 1995; 155: 2564-2570.

65 Fearon ER et al. Interleukin-2 production by tumor cells bypasses $\mathrm{T}$ helper function in the generation of an antitumor response. Cell 1990; 60: 397-403.

66 Lanzavecchia A. Identifying strategies for immune intervention. Science 1993; 260: 937-944.

67 Dutcher JP et al. A phase II study of interleukin-2 and lymphokine-activated killer cells in patients with metastatic malignant melanoma. J Clin Oncol 1989; 7: 477-485.

68 Rosenberg SA et al. Use of tumor-infiltrating lymphocytes and interleukin-2 in the immunotherapy of patients with metastatic melanoma. A preliminary report. New Engl J Med 1988; 319: 1676-1680.

69 Gansbacher B et al. Interleukin 2 gene transfer into tumor cells abrogates tumorigenicity and induces protective immunity. J Exp Med 1990; 172: 1217-1224.

70 Karp SE et al. Cytokine secretion by genetically modified nonimmunogenic murine fibrosarcoma. Tumor inhibition by IL-2 but not tumor necrosis factor. I Immunol 1993; 150: 896-908.

71 Haddada $\mathrm{H}$ et al. Adenoviral interleukin-2 gene transfer into p815 tumor cells abrogates tumorigenicity and induces antitumoral immunity in mice. Hum Gene Ther 1993; 4: 703-711.

72 Addison CL et al. Intratumoral injection of an adenovirus expressing interleukin 2 induces regression and immunity in a murine breast cancer model. Proc Natl Acad Sci USA 1995; 92 $8522-8526$.

73 Chen SH et al. Combination gene therapy for liver metastasis of colon carcinoma in vivo. Proc Natl Acad Sci USA 1995; 92: 2577-2581.

74 Cordier L et al. Complete recovery of mice from a pre-established tumor by direct intratumoral delivery of an adenovirus vector harboring the murine IL-2 gene. Gene Therapy 1995; 2: $16-21$

75 Parker SE et al. Plasmid DNA gene therapy: studies with the human interleukin-2 gene in tumor cells in vitro and in the murine B16 melanoma model in vivo. Cancer Gene Ther 1996; 3 : 175-185. 
76 Qin H, Chatterjee SK. Recombinant vaccinia expressing interleukin-2 for cancer gene therapy. Cancer Gene Ther 1996; 3: 163-167.

77 Toloza EM et al. In vivo cancer gene therapy with a recombinant interleukin-2 adenovirus vector. Cancer Gene Ther 1996; 3: 11-17.

78 Hirschowitz EA et al. In vivo adenovirus-mediated gene transfer of the Escherichia coli cytosine deaminase gene to human colon carcinoma-derived tumors induces chemosensitivity to 5-fluorocytosine. Hum Gene Ther 1995; 6: 1055-1063.

79 Rosenfeld MA et al. In vivo transfer of the human cystic fibrosis transmembrane conductance regulator gene to the airway epithelium. Cell 1992; 68: 143-155.
80 Rosenfeld MA et al. Adenovirus-mediated transfer of a recombinant alpha 1-antitrypsin gene to the lung epithelium in vivo. Science 1991; 252: 431-434.

81 Thomson TM et al. Pigmentation-associated glycoprotein of human melanomas and melanocytes: definition with a mouse monoclonal antibody. J Invest Dermatol 1985; 85: 169-174.

82 Poste G, Fidler IJ. The pathogenesis of cancer metastasis. Nature 1980; 283: 139-146.

83 Bouchard B, Vijayasaradhi S, Houghton AN. Production and characterization of antibodies against human tyrosinase. J Invest Dermatol 1994; 102: 291-295. 